\title{
Bonding of different self-adhesive resins to high-strength composite resin blocks treated with surface conditioning
}

Type of article: original article

Abbreviated title: bonding to hybrid resin blocks

Keywords: micro-tensile bond strength; contact angle; SEM, EDS, surface roughness

Declarations of interest: none

Hao $\mathrm{Yu}^{1,2, *}$, Keiichi Yoshida ${ }^{3}$, Hui Cheng ${ }^{1}$, Takashi Sawase ${ }^{2}$

1 Department of Prosthodontics, School and Hospital of Stomatology, Fujian Medical University, China

2 Department of Applied Prosthodontics, Graduate School of Biomedical Sciences, Nagasaki University, Nagasaki, Japan

3 Clinic of Fixed Prosthodontics, Nagasaki University Hospital, Nagasaki, Japan

* Corresponding author at: Department of Prosthodontics, School and Hospital of Stomatology, Fujian Medical University, China. Fax: +86 591 83720599; E-mail address: haoyu-cn@hotmail.com $(\mathrm{H} . \mathrm{Yu})$

Number of pages: 24

Number of tables: 6

Number of figures: 2

Quantity of reprints desired: 0 


\title{
Bonding of different self-adhesive resins to high-strength composite resin block treated with surface conditioning
}

\author{
Abstract \\ Purpose: This study aimed to evaluate the effects of chemical conditioning and self- \\ adhesive resins (SARs) on the bonding of mechanically conditioned high-strength \\ composite resin block (HSCRB). \\ Methods: Eighteen sections of HSCRB (KZR-CAD HR 3 Gammatheta, Yamakin) \\ were treated with alumina air abrasion and randomly divided into 3 groups according \\ to the SARs for bonding: RelyX Unicem 2 (RXU), SA Luting Plus (SAL), and G- \\ Cem ONE (GCO). The sections were further divided into 3 subgroups according to \\ the chemical conditioning of the adherend surfaces: no conditioning $(\mathrm{C})$, universal \\ adhesive (UA), and a mixture of $\gamma$-MPTS and 10-MDP (MM). After the surface \\ conditioning, the sections were cemented with the SARs. Each cemented section was \\ cut into 40 beams. Half of the beams were thermocycled $\left(4^{\circ} \mathrm{C} / 60^{\circ} \mathrm{C}, 10,000\right.$ cycles $)$. \\ The micro-tensile bond strength ( $\mu \mathrm{TBS}$ ) values were measured using a universal \\ testing machine. Scanning electron microscopy (SEM), energy-dispersive X-ray \\ spectroscopy (EDS), contact angles, and surface roughness measurements were \\ performed on the adherend surfaces of each subgroup. \\ Results: RXU showed the highest $\mu$ TBS values among the 3 SARs tested, while MM \\ application exhibited the highest $\mu$ TBS values among the 3 chemical conditioning \\ methods tested. After thermocycling, the samples in the RXU/MM, RXU/UA, and
}


GCO/MM groups showed no significant changes in the $\mu$ TBS values, whereas the others showed a significant reduction.

Conclusions: The bond strength of HSCRB was influenced by the chemical conditioning, SARs, and aging. $\gamma$-MPTS and 10-MDP application yielded higher $\mu$ TBS values of mechanically treated HSCRB than the UA. 


\section{Introduction}

The global evolution of computer-aided design/computer-aided manufacturing (CAD/CAM) technology led to the development of composite resin blocks (CRBs), which are designed to exhibit the characteristics of conventional ceramics and composites [1,2]. Currently, two types of CRBs based on microstructure exist: CRBs with dispersed fillers and polymer-infiltrated ceramic network (PICN) materials. With the aid of industrial fabrication with high temperature $\left(>100^{\circ} \mathrm{C}\right)$ and/or high pressure (>150 MPa), CRBs were shown to have superior material homogeneity and reliability compared to conventional composites [2]. Contemporary CRBs have a flexural strength ranging from 148.7 to $216.5 \mathrm{MPa}$ and a translucency similar to that of glass ceramics, rendering them an excellent material option for inlays, onlays and veneers $[3,4]$. On the other hand, resin-fixed prostheses are covered by Japanese social health insurance and have attracted substantial attention from both patients and clinicians $[5$, $6]$.

Adhesive cementation between resin and CRBs is a vital factor in the long-term success of restorations [7]. Schwenter et al. [8] reported that a similar shear bond strength of CRBs was achieved with self-adhesive resins (SARs) and conventional adhesive resins. Because SARs have much lower technical sensitivity, their use is clinically beneficial. Furthermore, since different SARs may exhibit different bonding properties depending on their composition, it is important to carry out a systematic investigation regarding their bonding performances to CRBs. Notably, the high degree of polymerization achieved by the industrial fabrication process may hinder 
bonding to CRBs $[9,10]$. Therefore, a wide range of surface conditioning methods have been proposed and evaluated by previous studies [4, 6, 11-13], and the choices of conditioning methods are dependent on the compositions of CRBs [14]. The literature seems to reach a consensus that the combination of mechanical conditioning and chemical conditioning prior to bonding yields the highest bond strength of CRBs $[4,11,13]$. More specifically, well-established mechanical conditioning (e.g., alumina air abrasion for CRBs with dispersed fillers and hydrofluoric acid etching for PICN materials) followed by silanization could be beneficial for the long-term bond strength of CRBs. The bonding mechanism may include: 1) micromechanical interlocking between CRBs and resins, or 2) chemical bonding established by silane coupling agents between the glassy phase of CRBs and resins $[4,13]$. Moreover, the wettability of the conditioned surface is important for bonding of CRBs. The silane coupling agents render the CRBs surface hydrophobic, and the non-hydrolyzable functional groups at the other end of the silane molecules can co-polymerize with the methacrylate groups of resins [15].

Recently, many so-called universal adhesives (UAs) have been developed and introduced into the dental market. UAs generally include a silane coupling agent, an acidic functional monomer (e.g., 10-methacryloyloxydecyl dihydrogen phosphate (10-MDP)), a solvent (e.g., ethanol, water), and/or a methacrylate monomer [16]. 10MDP has been demonstrated to promote bonding of zirconia, alumina, and metals, which are the components of CRBs $[17,18]$. Moreover, chemical bonding to the polymer may be achieved via acid groups of the copolymer and 10-MDP in the UAs 
[13]. In this manner, the UAs may establish adhesion to both the polymer and glassy phases of CRBs and provide better performance than that provided by silane coupling agents alone. In a recently published study, UA application without mechanical conditioning showed significantly higher bond strength values of PICN material than those resulting from the other mechanical conditioning methods (alumina air abrasion, Er,Cr:YSGG laser treatment, and hydrofluoric acid etching) [19]. However, limited information is available to compare the effects of UAs and silane coupling agents on CRBs.

Recently, high strength CRBs (HSCRBs, e.g., KZR-CAD HR3 Gammatheta, Yamakin, Kouchi, Japan) were developed and introduced into the market. The HSCRBs were claimed to have a higher flexural strength (approximately $300 \mathrm{MPa}$ according to the manufacturer's internal data) than that of conventional CRBs [20]. Moreover, the fluoride release from fluoridated fillers may inhibit the bacterial adhesion and maintain surface integrity during its long-term service. The abovementioned properties make it a good alternative to its competitor on the market. According to the manufacturer's recommendation, this material needs to be treated with alumina air abrasion and chemical conditioning prior to bonding [20]. Other than the manufacturer's internal data, information concerning this novel material is very limited. Thus, the objective of this investigation was to evaluate the effects of different chemical conditioning methods following alumina air abrasion on the microtensile bond strength ( $\mu$ TBS) of SARs to the HSCRB. The following null hypotheses were tested: 1) that no differences exist among the 3 chemical conditioning methods 
in terms of bond strength, surface morphology, surface composition, and surface roughness to the HSCRB; 2) that no differences exist among the 3 SARs in terms of bond strength to the HSCRB; and 3) that the bond strength of the HSCRB remains the same after thermocycling. 


\section{Materials and methods}

One HSCRB (KZR-CAD HR 3 Gammatheta, Yamakin, Kouchi, Japan [KC]) and 3 dual-cure SARs (RelyX Unicem 2, 3M ESPE, St. Paul, MN, USA [RXU]; SA Luting Plus, Kuraray Noritake Dental, Tainai, Japan [SAL]; and G-Cem ONE, GC Corp., Tokyo, Japan [GCO]) were investigated in this study (Table 1).

\subsection{Surface conditioning}

Eighteen sections $(14.5 \times 14.5 \times 6 \mathrm{~mm})$ were obtained from $\mathrm{KC}$ blocks using a low-speed diamond saw (Isomet, Buehler, Lake Bluff, IL, USA) under continuous water cooling. The sections were randomly divided into 3 groups according to the SARs used for bonding. The cementation surfaces of the sections were wet-polished with 600-grit silicone-carbide abrasive paper using a polishing machine (ML-160A, Maruto, Musashino, Japan) under continuous water cooling. They were sandblasted with $50-\mu \mathrm{m}$ aluminum oxide particles (Hi Aluminas, Shofu, Kyoto, Japan) at $0.2 \mathrm{MPa}$ for $15 \mathrm{~s}$ at a distance of $10 \mathrm{~mm}$ using a sandblasting device (Jet Blast III, J. Morita, Kyoto, Japan). The specimens were cleaned with phosphoric acid (K-etchant GEL, Kuraray Noritake Dental, Tainai, Japan) for $5 \mathrm{~s}$ and then ultrasonically cleaned in distilled water for $1 \mathrm{~min}$. After cleaning, the specimens were air dried using a 3-way syringe for $10 \mathrm{~s}$. Each group was further divided into 3 subgroups according to the chemical conditioning performed on the cementation surfaces ( 2 sections per subgroup).

Subgroup C (control): no chemical conditioning was performed. 
Subgroup UA (universal adhesive): the UA (Bondmer Lightless, Tokuyama Dental, Ibaraki, Japan) was freshly mixed, applied to the cementation surfaces, and then air dried using a 3-way syringe for $5 \mathrm{~s}$.

Subgroup MM (MPTS + MDP): a mixture of $\gamma$-methacryloyloxypropyl

trimethoxysilane ( $\gamma$-MPTS) and 10-MDP in equal volumes was freshly prepared, applied to the cementation surfaces, and then air dried using a 3-way syringe for $5 \mathrm{~s}$.

\subsection{Cementation procedure}

After surface conditioning, 2 sections in the same group (with the same surface conditioning) were cemented together using 1 of the 3 dual-cure SARs tested. Before applying the SARs, two pieces of 2-mm-wide polyethylene adhesive tape (50 $\mu \mathrm{m}$ thick) were attached at the margins of 1 cementation surface as a spacer to standardize the bonding agent/luting cement thickness at $50 \mu \mathrm{m}$. The cement was left to set primarily in self-cure mode for the first $2 \mathrm{~min}$ at ambient room light, thereby simulating the clinical time needed for cementation prior to actual light curing [21]. Light irradiation was then performed by placing the tip of the LED light-curing unit (power density of $1000 \mathrm{~mW} / \mathrm{cm}^{2}$; Pen-cure, J. Morita, Osaka, Japan) on 4 sides for a total of $160 \mathrm{~s}$. The bonded sections were stored in distilled water at $37^{\circ} \mathrm{C}$ for $24 \mathrm{~h}$ prior to specimen preparation for the $\mu \mathrm{TBS}$ test.

\subsection{Micro-tensile bond strength test}

Forty beams (bonding area: $\sim 0.81 \mathrm{~mm}^{2}$ ) were cut from each bonded section using a low-speed diamond saw under constant water cooling. The exact bonded area of each 
beam was measured with a digital caliper (Mitutoyo, Tokyo, Japan) and further used for the $\mu$ TBS calculation.

Per chemical conditioning and per SAR, 40 beams were prepared and tested under 2 different storage conditions ( $\mathrm{n}=20$ per storage condition). Non-aged beams were stored in distilled water at $37^{\circ} \mathrm{C}$ for $24 \mathrm{~h}$ prior to the $\mu \mathrm{TBS}$ test. The aged beams were subjected to 10,000 thermal cycles between water baths (Rika-Kogyo, Tokyo, Japan) held at $4^{\circ} \mathrm{C}$ and $60^{\circ} \mathrm{C}$, with a dwelling time of 1 min per cycle in each bath $[22,23]$.

Following storage, the ends of each beam were fixed to a jig with cyanoacrylate glue (Model Repair, Dentsply-Sankin, Tokyo, Japan). The jig was fixed into a universal testing machine (EZ-test short, Shimadzu, Kyoto, Japan) and stressed under tensile force at a crosshead speed of $1 \mathrm{~mm} / \mathrm{min}$ until failure occurred. The $\mu \mathrm{TBS}$ (in $\mathrm{MPa}$ ) was then calculated by dividing the imposed force (in N) at the time of fracture by the bonded area (in $\mathrm{mm}^{2}$ ). Pre-test failures were recorded when the specimens failed before the actual testing.

The debonded surfaces of all specimens were examined using an optical microscope (SMZ-10, Nikon, Tokyo, Japan) at a magnification of 50x. The mode of failure was categorized as adhesive failure (at the CRB/SAR interface), cohesive failure (within the CRB/SAR), or mixed failure (combination of the two modes on the same surface).

\subsection{Contact angle measurement of adherend surfaces}

The difference in the hydrophilicity/hydrophobicity of the KC material after surface conditioning was determined by the contact angle measurements, obtained 
with a contact angle goniometer (CA-D, Kyowa Interface Science, Niiza, Japan). Distilled water was used as the test liquid. The measurements were performed on the conditioned specimen surfaces using the sessile-drop method at $23 \pm 1^{\circ} \mathrm{C}$ before and after ethanol cleaning. Briefly, ethanol cleaning was performed by placing the specimens in absolute ethanol solvent in the ultrasonic bath for 2 min to remove any non-chemically-bonded molecules after chemical conditioning [24]. Five sets of contact angle measurements were obtained for each sample, and the right and left contact angles for the droplets were averaged. The size and volume of the drops were kept constant, as variations in the drop volume can lead to inconsistent contact angle measurements [25]. Final contact angle measurements were recorded (usually within $10-15 \mathrm{~s})$ once their values stabilized after drop placement.

\subsection{Surface roughness measurement of adherend surfaces}

After surface conditioning, the surface roughness of the KC specimens ( $\mathrm{n}=8$ per chemical conditioning) was measured using a surface profilometer (SEF680, Kosaka, Tokyo, Japan). For each measurement, the surface roughness was measured from the profiles obtained by a needle passing across a length of $6 \mathrm{~mm}$ at a speed of $0.5 \mathrm{~mm} / \mathrm{s}$. The average arithmetical mean roughness $(\mathrm{Ra})$ values $(\mu \mathrm{m})$ were calculated.

\subsection{Scanning electron microscopy (SEM) observation and energy-dispersive $X$ -}

\section{ray spectroscopy (EDS) analysis of adherend surfaces}

After surface conditioning, $\mathrm{KC}$ specimens ( $\mathrm{n}=3$ per chemical conditioning) were analyzed by scanning electron microscopy (SEM) (Quanta 250, FEI, Hillsboro, OR, USA). The specimens were mounted on aluminum stubs and sputter-coated with gold 
before being examined at an acceleration voltage of $15 \mathrm{kV}$. Furthermore, quantitative changes in the adherend surface composition were evaluated using EDS. The respective EDS spectra were obtained over a $400 \times 400 \mu \mathrm{m}$ area with an acceleration voltage of $15 \mathrm{kV}$.

\subsection{Statistical analysis}

The assumption of normality and equality of variances was confirmed with the Shapiro-Wilk and Levene tests, respectively. The $\mu$ TBS data were analyzed using three-way analysis of variance (ANOVA), the contact angle data were analyzed using two-way ANOVA, and the surface roughness data and EDS data were analyzed using one-way ANOVA. Non-parametric failure mode data were analyzed using Fisher's exact probability test. All statistical analyses were performed using PASW version 18 (SPSS, Chicago, IL, USA) at a significance level of 5\%. 


\section{Results}

\subsection{SEM and EDS results of adherend surfaces}

Representative SEM images are shown in Figure 1. Similar surface morphology was found in the subgroups C (control) and MM (MPTS + MDP). In contrast, the surface of subgroup UA (universal adhesive) was covered by a thin film (Fig. 1). Table 2 presents the concentration (wt $\%$ ) of the respective elements on the adherend surfaces as detected by EDS. In particular, after surface conditioning with UA (1.23 \pm $0.35 \mathrm{wt} \%)$ and MPTS + MDP $(1.31 \pm 0.32 \mathrm{wt} \%)$, significant increases in P concentration were found on the surface that corresponds to phosphate.

\subsection{Surface roughness measurement of adherend surfaces}

The Ra values of all tested adherend surfaces ranged from 2.20 to $3.10 \mu \mathrm{m}$ (Table 3). Significant effects were found for the factor "chemical conditioning" $(P<0.001)$. Subgroup UA $(3.10 \pm 0.35 \mu \mathrm{m})$ has demonstrated the highest Ra values, followed by subgroups MM $(2.81 \pm 0.17 \mu \mathrm{m})$ and $\mathrm{C}(2.20 \pm 0.11 \mu \mathrm{m})$.

\subsection{Contact angle measurement of adherend surfaces}

Significant effects were found for the factors "chemical conditioning" and "ethanol cleaning" (all $P<0.001$ ). Furthermore, there was a significant interaction between chemical conditioning and ethanol cleaning $(P<0.001)$. The initial water contact angle (before ethanol cleaning) of $\mathrm{KC}$ material ranged from $28.42^{\circ}$ to $56.16^{\circ}$ (Table 4). After ethanol cleaning, the samples from the subgroups UA and MM showed an increase in the contact angle, whereas no changes were found in subgroup C. The 
samples from subgroup MM yielded significantly higher water contact angles than those from subgroups UA and C.

\subsection{Micro-tensile bond strength test and failure mode analysis}

No pre-test failures were observed in the present study. According to the three-way ANOVA of the $\mu$ TBS data, significant effects were found for the factors "cement system", "chemical conditioning", and "aging" (Table 5). There were significant twofactor interactions between the cement system and chemical conditioning $(P<0.001)$ as well as between chemical conditioning and aging $(P<0.001)$. However, the interaction between the cement system and aging was not significant $(P=0.083)$. Moreover, there was a significant three-factor interaction among the cement system, chemical conditioning and aging $(P<0.001)$.

The means and standard deviations of the $\mu$ TBS values are listed in Table 6 . The $\mu$ TBS values in the RXU group were significantly higher than those in the other 2 groups. The $\mu$ TBS values of the tested SARs can be ranked as follows: RXU > GCO $>$ SAL. Chemical conditioning following alumina air abrasion prior to bonding with the SARs yielded significantly higher $\mu$ TBS values than those of the control under both non-aged and aged conditions. MM application prior to bonding yielded the highest bond strength among the different chemical conditioning methods used. The $\mu$ TBS values of the tested chemical conditioning methods can be ranked as follows: $\mathrm{MM}>\mathrm{UA}>\mathrm{C}$. After thermocycling, the samples in the RXU/MM, RXU/UA, and GCO/MM groups showed no significant changes in the $\mu \mathrm{TBS}$ values, whereas the others showed a significant reduction. 
The failure mode distribution of the different groups is shown in Fig. 2. The predominant failure mode was cohesive failure in all groups. Overall, both chemical conditioning and aging showed a significant effect on the fractography $(P=0.005$ and $P<0.001$, respectively). The cement system had no influence on the failure mode ( $P$ $=0.791)$. Increased adhesive failures were observed in all groups after aging, while chemical conditioning led to fewer adhesive failures. 


\section{Discussion}

Based on the present findings, the null hypotheses that no differences existed among the 3 chemical conditioning methods and among the 3 SARs in terms of bond strength, surface morphology, surface composition, and surface roughness to HSCRB were rejected. The null hypothesis that the bond strength of HSCRB would remain the same after thermocycling was accepted for the RXU/MM, RXU/UA, and GCO/MM groups.

Silane coupling agents are useful and effective in promoting adhesion between resin and silica-based restorative materials, such as glass ceramic and CRBs [26]. After being activated in an acidic environment, the silane coupling agent undergoes the hydrolysis process and forms strong siloxane (-Si-O-Si-) linkages on the silicabased material surface [27]. Recently, it has been found that the bond strength of the most frequently used silane coupling agent ( $\gamma$-MPTS) activated by the acidic functional monomer (10-MDP) was approximately two-fold greater than those obtained with acetic acid [28]. When hydrolyzed with 10-MDP, the self-dehydration condensation of $\gamma$-MPTS could be inhibited, thereby resulting in an increased number of silane molecules bonded to the glassy phases and a higher bond strength. Thus, the increase of Si on EDS was revealed in both subgroups UA and MM, although the increase was not significant (Table 2). Furthermore, 10-MDP is a proven functional adhesive monomer that can chemically bond to zirconia, alumina, and metals [29]. In terms of effects, both subgroups UA and MM revealed an increased wt $\%$ in $\mathrm{P}$. The increase of both Si and P on EDS indicates the synergistic effects of $\gamma$-MPTS and 10- 
MDP, which improve the bonding performances of HSCRB. Therefore, both the UA and MM applications yielded a significant increase in the bond strength and fewer adhesive failures than the controls. A high prevalence of cohesive and mixed failures also implied a strong bond to the conditioned HSCRB.

Interestingly, the bond strength of $\mathrm{KC}$ material differed between the commercial UA (Bondmer Lightless, Tokuyama Dental) and the mixture of $\gamma$-MPTS and 10MDP. Specifically, in contrast to the samples treated with the UA, the samples treated with the mixture of $\gamma$-MPTS and 10-MDP exhibited significantly higher $\mu$ TBS values. The UA tested contains $\gamma$-MPTS and 10-MDP, as do most commercially available products on the market. Previous studies showed that the $\gamma$-MPTS in the UA may become hydrolyzed and deteriorate in a single bottle due to the presence of water with 10-MDP and may not be effective in optimizing bonding to silica-based restorative materials $[16,30]$. Moreover, the residual solvent (water) that remains within the adhesive interface may weaken the bond [31]. However, this may not be the case for the UA application since the $\gamma$-MPTS and 10-MDP were stored in separate bottles and were mixed immediately before application to the HSCRB surface. The difference in the performances of the UA and the mixture of $\gamma$-MPTS and 10-MDP may be related to the following factors: 1 ) the different effects of the UA and the mixture of $\gamma$-MPTS and 10-MDP on the chemical functionalization of the adherend surfaces. Greater effects were associated with the application of $\gamma$-MPTS and 10-MDP since the increased surface P concentration was detected by EDS analysis. Only a minor increase in the surface Si concentration was found after surface conditioning, possibly 
due to the Si-rich surface of the KC block; 2) as a self-assembled ultra-thin film, the $\gamma$-MPTS and 10-MDP coating has a weaker mechanical strength than SAR and HSCRB and can be regarded as the weakest point in the adhesion system [32]. An increase in the coating thickness, as observed on the adherend surfaces after the UA application, may reduce the bonding performance of the chemical conditioning [33]; or 3) organic additives in the UA and the different concentration of $\gamma$-MPTS and 10MDP in the 2 solutions tested may also play an important role $[28,34]$. In addition, the samples treated with the UA exhibited different initial contact angles (before ethanol rinsing) than those treated with the mixture of $\gamma$-MPTS and 10-MDP. This result clearly demonstrated that the organic additives in the UA are deposited and/or physiosorbed onto the HSCRB surface. Ethanol cleaning removed the non-chemically bonded molecules and therefore led to an increase in the contact angle [24]. The observed lower contact angles with the UA probably indicate a decrease in the amount of chemisorbed $\gamma$-MPTS. This could be because the organic additives prevent chemisorption of $\gamma$-MPTS [28]. It is also important to note that slightly rougher surfaces were found after the application of UA and the mixture of $\gamma$-MPTS and 10MDP. The changes in surface roughness may affect the surface wettability and contribute to the enhanced bonding performance of the adherend surfaces [35].

The surface of subgroup UA was rougher than that of other subgroups. It should be noted that the UA contains an unknown amount of methacrylates such as Bis-GMA, TEGDMA and HEMA. These methacrylates can adhere to any resin-based dental restorative materials as well as primer (e.g. MTU-6, $\gamma$-MPTS or 10-MDP) treated 
surfaces. These methacrylates contain $\mathrm{C}=\mathrm{C}$ and polymerizable to become polymer under certain conditions, such as exposure to an acryl borate catalyst, which is present in liquid B of the UA. Therefore, thicker film layers were revealed by SEM (Fig. 1 C and D), which contribute to the roughness on the substrate surface [36].

The bond strength and failure mode differed between the non-aged and aged samples. The non-aged samples exhibited significantly higher $\mu$ TBS values and predominantly suffered cohesive failure. Temperature changes during the thermocycling process may amplify the coefficient of thermal expansion mismatch of the bonded materials, which generates mechanical stresses at the bonded interface, resulting in bonding degradation [37]. Notably, the bond strength of the $\mathrm{KC}$ material treated with the mixture of 10-MDP and $\gamma$-MPTS and followed by cementation with RXU and GCO remained stable after thermocycling. From a clinical viewpoint, treating the KC materials with alumina air abrasion and a mixture of $\gamma$-MPTS and 10MDP may be advantageous to provide long-term adhesion.

Although there are a wide variety of dental materials available for CAD/CAM restorations, HSCRBs have been proven adequate for veneers, inlays/onlays, and crowns due to their superior aesthetic and mechanical properties [1, 2]. However, bonding to HSCRBs is still challenging. This systematic investigation was carried out to determine the effects of chemical conditioning and SARs on the bond strength of mechanically conditioned HSCRB. It has been recommended that micro-retentive surfaces should be generated by either blasting or hydrofluoric acid etching to improve the bonding properties of CRBs [7, 38]. Therefore, the surfaces of HSCRB 
were all treated with alumina air abrasion prior to any chemical conditioning to simulate clinical situations and to adhere to the manufacturer's instructions.

Limited information is available regarding the effects of SARs on the bond strengths of HSCRBs. Of the 3 SARs tested, RXU showed the highest bond strength to HSCRB, which is in accordance with previous studies $[39,40]$. This phenomenon may be explained by the fact that RXU has higher filler loading (72 wt\%) and larger filler particle sizes than the other SARs tested, which leads to superior mechanical properties of the cement and, thus, stronger engagement of the interlocking on the HSCRB surface treated with alumina oxide particles [40]. Moreover, the superior $\mathrm{pH}-$ neutralization behavior of RXU may have a positive effect on its mechanical stability during thermocycling [41].

Although this in vitro study cannot exactly replicate the intraoral conditions with all individual variations, it provides some insights into the effects of chemical conditioning and SARs on the bonding properties of HSCRB. However, the findings should be interpreted with caution before being applied to clinical situations. Future laboratory and clinical studies are needed to confirm the long-term bonding performance and provide evidence-based guidelines for clinical practice. 


\section{Conclusion}

Within the limitations of this study, the following conclusions can be drawn:

(1) Chemical conditioning with the universal adhesive and a mixture of $\gamma$-MPTS and 10-MDP increased the bond strength of HSCRB.

(2) The bond strength varied among the different SARs. RelyX Unicem 2 showed significantly higher $\mu$ TBS values than SA Luting Plus and G-Cem ONE.

(3) Thermocycling influenced the bond strength of SARs to HSCRBs, while the bond strength was maintained when HSCRB were treated with alumina air abrasion followed by an application of a mixture of $\gamma$-MPTS and 10-MDP. 


\section{Conflict of interest}

The authors declare no conflict of interest. 


\section{References}

[1] Ruse ND, Sadoun MJ. Resin-composite blocks for dental CAD/CAM applications. J Dent Res 2014; 93:1232-4.

[2] Mainjot AK, Dupont NM, Oudkerk JC, Dewael TY, Sadoun MJ. From artisanal to CAD-CAM blocks: state of the art of indirect composites. J Dent Res 2016; 95:487-95.

[3] Awada A, Nathanson D. Mechanical properties of resin-ceramic CAD/CAM restorative materials. J Prosthet Dent 2015; 114:587-93.

[4] El-Damanhoury HM, Gaintantzopoulou MD. Self-etching ceramic primer versus hydrofluoric acid etching: etching efficacy and bonding performance. J Prosthodont Res 2018; 62:75-83.

[5] Higashi M, Matsumoto M, Kawaguchi A, Miura J, Minamino T, Kabetani T, et al. Bonding effectiveness of self-adhesive and conventional-type adhesive resin cements to $\mathrm{CAD} / \mathrm{CAM}$ resin blocks. part 1: effects of sandblasting and silanization. Dent Mater J 2016; 35:21-8.

[6] Kawaguchi A, Matsumoto M, Higashi M, Miura J, Minamino T, Kabetani T, et al. Bonding effectiveness of self-adhesive and conventional-type adhesive resin cements to $\mathrm{CAD} / \mathrm{CAM}$ resin blocks. part 2: effect of ultrasonic and acid cleaning. Dent Mater J $2016 ; 35: 29-36$

[7] Spitznagel FA, Horvath SD, Guess PC, Blatz MB. Resin bond to indirect composite and new ceramic/polymer materials: a review of the literature. J Esthet Restor Dent $2014 ; 26: 382-93$.

[8] Schwenter J, Schmidli F, Weiger R, Fischer J. Adhesive bonding to polymer 
infiltrated ceramic. Dent Mater J 2016; 35:796-802.

[9] Bähr N, Keul C, Edelhoff D, Eichberger M, Roos M, Gernet W, et al. Effect of different adhesives combined with two resin composite cements on shear bond strength to polymeric CAD/CAM materials. Dent Mater J 2013; 32:492-501.

[10] Spitznagel FA, Vuck A, Gierthmühlen PC, Blatz MB, Horvath SD. Adhesive bonding to hybrid materials: an overview of materials and recommendations. Compend Contin Educ Dent 2016; 37:630-7.

[11] Frankenberger R, Hartmann VE, Krech M, Kramer N, Reich S, Braun A, et al. Adhesive luting of new CAD/CAM materials. Int J Comput Dent 2015; 18:9-20.

[12] Cekic-Nagas I, Ergun G, Egilmez F, Vallittu PK, Lassila LV. Micro-shear bond strength of different resin cements to ceramic/glass-polymer CAD-CAM block materials. J Prosthodont Res 2016; 60:265-73.

[13] Rohr N, Flury A, Fischer J. Efficacy of a universal adhesive in the bond strength of composite cements to polymer-infiltrated ceramic. J Adhes Dent 2017; 19:417-24. [14] Campos F, Almeida CS, Rippe MP, de Melo RM, Valandro LF, Bottino MA. Resin bonding to a hybrid ceramic: effects of durface treatments and aging. Oper Dent 2016; $41: 171-8$

[15] Matinlinna JP, Vallittu PK. Bonding of resin composites to etchable ceramic surfaces - an insight review of the chemical aspects on surface conditioning. J Oral Rehabil 2007; 34:622-30.

[16] Noda Y, Nakajima M, Takahashi M, Mamanee T, Hosaka K, Takagaki T, et al. The effect of five kinds of surface treatment agents on the bond strength to various ceramics 
with thermocycle aging. Dent Mater J 2017; 36:755-61.

[17] Agingu C, Zhang C, Jiang N, Cheng H, Özcan M, Yu H. Intraoral repair of chipped or fractured veneered zirconia crowns and fixed dental prosthesis: clinical guidelines based on literature review. J Adhes Sci Technol 2018; 32:1711-23.

[18] Yagawa S, Komine F, Fushiki R, Kubochi K, Kimura F, Matsumura H. Effect of priming agents on shear bond strengths of resin-based luting agents to a translucent zirconia material. J Prosthodont Res 2018; 62:204-9.

[19] Barutcigil K, Barutcigil Ç, Kul E, Özarslan MM, Buyukkaplan US. Effect of different surface treatments on bond dtrength of resin cement to a CAD/CAM restorative material. J Prosthodont 2016. doi: 10.1111/jopr.12574.

[20] Yamakin. KZR-CAD HR3 Gammatheta Instruction Sheets, http://www.yamakingold.co.jp/technical_support/webrequest/pdf/kzr-cad_hrb3_1802.pdf; 2018 [accessed 12 June 2018].

[21] Peumans M, Valjakova EB, De Munck J, Mishevska CB, Van Meerbeek B. Bonding effectiveness of luting composites to different CAD/CAM materials. J Adhes Dent 2016; 18:289-302.

[22] Shinohara A, Taira Y, Sawase T. Effects of tributylborane-activated adhesive and two silane agents on bonding computer-aided design and manufacturing (CAD/CAM) resin composite. Odontology 2017; 105:437-42.

[23] Nobuaki A, Keiichi Y, Takashi S. Effects of air abrasion with alumina or glass beads on surface characteristics of CAD/CAM composite materials and the bond strength of resin cements. J Appl Oral Sci 2015; 23:629-36. 
[24] Arai M, Takagaki T, Takahashi A, Tagami J. The role of functional phosphoric acid ester monomers in the surface treatment of yttria-stabilized tetragonal zirconia polycrystals. Dent Mater J 2017; 36:190-4.

[25] Faibish SR, Yoshida W, Cohen Y. Contact angle study on polymer-grafted silicon Wafers. J Colloid Interface Sci 2002; 256:341-50.

[26] Matinlinna JP, Lung CYK, Tsoi JKH. Silane adhesion mechanism in dental applications and surface treatments: a review. Dent Mater 2018; 34:13-28.

[27] Karabela MM, Sideridou ID. Effect of the structure of silane coupling agent on sorption characteristics of solvents by dental resin-nanocomposites. Dent Mater 2008; 24:1631-9.

[28] Maruo Y, Nishigawa G, Yoshihara K, Minagi S, Matsumoto T, Irie M. Does 8methacryloxyoctyl trimethoxy silane (8-MOTS) improve initial bond strength on lithium disilicate glass ceramic? Dent Mater 2017; 33:e95-e100.

[29] Chuang SF, Kang LL, Liu YC, Lin JC, Wang CC, Chen HM, et al. Effects of silaneand MDP-based primers application orders on zirconia-resin adhesion-a ToF-SIMS study. Dent Mater 2017; 33:923-33.

[30] Taguchi S, Komine F, Kubochi K, Fushiki R, Kimura F, Matsumura H. Effect of a silane and phosphate functional monomer on shear bond strength of a resin-based luting agent to lithium disilicate ceramic and quartz materials. J Oral Sci 2018; 60:360-6.

[31] Van Meerbeek B, De Munck J, Yoshida Y, Inoue S, Vargas M, Vijay P, et al. Buonocore memorial lecture. Adhesion to enamel and dentin: current status and future challenges. Oper Dent 2003; 28:215-35. 
[32] Queiroz JR, Benetti P, Ozcan M, de Oliveira LF, Della Bona A, Takahashi FE, et al. Surface characterization of feldspathic ceramic using ATR FT-IR and ellipsometry after various silanization protocols. Dent Mater 2012; 28:189-96.

[33] Hooshmand T, van Noort R, Keshvad A. Bond durability of the resin-bonded and silane treated ceramic surface. Dent Mater 2002; 18:179-88.

[34] Aida M, Tabei N, Kimoto S, Tanimura H, Takahashi H, Yaguchi T, et al. Ceramic bond durability and degradation mechanism of commercial gammamethacryloxypropyl trimethoxysilane-based ceramic primers. Am J Dent 2012; 25:231-4.

[35] Kawaguchi-Uemura A, Mine A, Matsumoto M, Tajiri Y, Higashi M, Kabetani T, et al. Adhesion procedure for $\mathrm{CAD} / \mathrm{CAM}$ resin crown bonding: reduction of bond strengths due to artificial saliva contamination. J Prosthodont Res 2018; 62:177-83.

[36] Giannini M, Paulillo LA, Ambrosano GM. Effect of surface roughness on amalgam repair using adhesive systems. Braz Dent J 2002; 13:179-83.

[37] Gale MS, Darvell BW. Thermal cycling procedures for laboratory testing of dental restorations. J Dent 1999; 27:89-99.

[38] Mine A, Kabetani T, Kawaguchi-Uemura A, Higashi M, Tajiri Y, Hagino R, et al. Effectiveness of current adhesive systems when bonding to $\mathrm{CAD} / \mathrm{CAM}$ indirect resin materials: a review of 32 publications. Jpn Dent Sci Rev. 2018. doi 10.1016/j.jdsr.2018.10.001

[39] Schwenter J, Schmidli F, Weiger R, Fischer J. Adhesive bonding to polymer infiltrated ceramic. Dent Mater J 2016; 35:796-802. 
[40] Kim JY, Cho GY, Roh BD, Shin Y. Effect of curing mode on shear bond strength of self-adhesive cement to composite blocks. Materials (Basel) 2016; 9:E210.

[41] Zorzin J, Petschelt A, Ebert J, Lohbauer U. pH neutralization and influence on mechanical strength in self-adhesive resin luting agents. Dent Mater 2012; 28:672-9. 
Table 1 Materials tested in this study

\begin{tabular}{llll}
\hline Material & Product & Manufacturer & Composition \\
\hline $\begin{array}{l}\text { Composite } \\
\text { resin blocks }\end{array}$ & $\begin{array}{l}\text { KZR-CAD HR } 3 \\
\text { Gammatheta }\end{array}$ & $\begin{array}{l}\text { Yamakin, Kouchi, } \\
\text { Japan }\end{array}$ & Methacrylate-based matrix, inorganic filler, pigment \\
& RelyX Unicem 2 & 3M ESPE, St. & Base paste: methacrylate monomers containing phosphoric acid groups, \\
& Paul, MN, USA & Catalyst paste: methacrylate monomers, alkaline (basic) fillers, silanated fillers, \\
& & & initiator components, stabilizers, pigments
\end{tabular}




\begin{tabular}{|c|c|c|c|}
\hline Universal & Bondmer Lightless & Tokuyama Dental, & Liquid A: phosphate monomer, Bis-GMA, TEGDMA, HEMA, MTU-6 \\
\hline adhesive & & Ibaraki, Japan & Liquid B: acetone, isopropanol, water, acryl borate catalyst, $\gamma$-MPTS, peroxide \\
\hline $\begin{array}{l}\text { 10-MDP } \\
\text { solution }\end{array}$ & 10-MDP solution & $\begin{array}{l}\text { GC Corp., Tokyo, } \\
\text { Japan }\end{array}$ & $5 \mathrm{wt} \% 10-\mathrm{MDP}$ in ethanol \\
\hline $\begin{array}{l}\text { Silane } \\
\text { solution }\end{array}$ & $\gamma$-MPTS solution & $\begin{array}{l}\text { Tokyo Chemical } \\
\text { Industry Co., Ltd, } \\
\text { Tokyo, Japan }\end{array}$ & $5 \mathrm{wt} \% \gamma$-MPTS in ethanol \\
\hline
\end{tabular}


Table 2 Means and standard deviations of the concentration (wt\%) of the elements in the different groups determined from EDS analysis $(400 \times 400 \mu \mathrm{m})$

\begin{tabular}{|c|c|c|c|c|c|c|c|c|c|c|}
\hline Surface conditioning & $\mathrm{C}$ & $\mathrm{O}$ & $\mathrm{F}$ & $\mathrm{Na}$ & $\mathrm{Al}$ & $\mathrm{Si}$ & $\mathrm{Sr}$ & $\mathrm{Ca}$ & $\mathrm{P}$ & $\mathrm{Cl}$ \\
\hline \multirow{2}{*}{ No conditioning } & 24.86 & 44.31 & 4.135 & 0.83 & 6.50 & 11.68 & 7.69 & & & \\
\hline & $(1.06) \mathrm{a}$ & $(1.00) \mathrm{a}$ & $(0.56) \mathrm{a}$ & $(0.22) \mathrm{a}$ & $(0.83) \mathrm{a}$ & $(1.24) \mathrm{a}$ & $(0.77) \mathrm{a}$ & - & - & - \\
\hline \multirow{2}{*}{ Universal adhesive } & 43.67 & 39.82 & 0.41 & 0.50 & 0.18 & 12.68 & 0.63 & 0.66 & 1.23 & 0.25 \\
\hline & $(0.47) b$ & $(2.97) b$ & $(0.57) b$ & $(0.08) b$ & $(0.04) b$ & $(1.34) \mathrm{a}$ & $(0.23) b$ & $(0.06) \mathrm{a}$ & $(0.35) \mathrm{a}$ & $(0.03) \mathrm{a}$ \\
\hline \multirow{2}{*}{$\gamma$-MPTS + 10-MDP } & 30.98 & 38.96 & 3.01 & 0.77 & 4.19 & 12.69 & 8.14 & & 1.31 & \\
\hline & $(4.65) \mathrm{c}$ & $(1.87) \mathrm{b}$ & $(0.57) \mathrm{c}$ & $(0.01) \mathrm{a}$ & $(0.74) \mathrm{c}$ & $(0.57) \mathrm{a}$ & $(0.88) \mathrm{a}$ & - & $(0.32) \mathrm{a}$ & - \\
\hline
\end{tabular}

Values marked with same lower letter were not significantly different within the column.

"-" = The concentration of the element was below the detection limit. 
Table 3 Means and standard deviations of the surface roughness $(\mu \mathrm{m})$ in the different groups

Surface conditioning

Ra values

No conditioning

$2.20(0.11) \mathrm{a}$

$\gamma$-MPTS + 10-MDP

$2.81(0.17) b$

Universal adhesive

$3.10(0.35) \mathrm{c}$

Values marked with the same lower-case letter were not significantly different within the column. 
Table 4 Means and standard deviations of the water contact angles $\left(^{\circ}\right)$ in the different groups

\begin{tabular}{lcc} 
Surface conditioning & Before ethanol cleaning & After ethanol cleaning \\
\hline No conditioning & $56.16(4.43) \mathrm{A}, \mathrm{a}$ & $55.94(4.15) \mathrm{A}, \mathrm{a}$ \\
Universal adhesive & $52.84(3.48) \mathrm{A}, \mathrm{a}$ & $64.73(4.16) \mathrm{B}, \mathrm{b}$ \\
$\gamma$-MPTS + 10-MDP & $28.42(3.04) \mathrm{A}, \mathrm{b}$ & $72.55(5.08) \mathrm{B}, \mathrm{c}$
\end{tabular}

Values marked with the same upper-case letter were not significantly different within each row; values marked with the same lower-case letter were not significantly different within each column. 
Table 5 Three-way analysis of $\mu$ TBS values

\begin{tabular}{|c|c|c|c|c|c|}
\hline & Type III sum & & Mean & & \\
\hline Source & of squares & $d f$ & square & $\mathrm{F}$ & $P$ \\
\hline Cement system & 10214.358 & 2 & 5107.179 & 42.066 & $<0.001$ \\
\hline Chemical conditioning & 41579.034 & 2 & 20789.517 & 171.236 & $<0.001$ \\
\hline Aging & 15142.574 & 1 & 15142.574 & 124.724 & $<0.001$ \\
\hline \multicolumn{6}{|l|}{ Cement system X Chemical } \\
\hline conditioning & 3399.432 & 4 & 849.858 & 7.0000 & $<0.001$ \\
\hline Cement system X Aging & 610.000 & 2 & 305.000 & 2.512 & 0.083 \\
\hline \multicolumn{6}{|c|}{ Cement system X Chemical } \\
\hline conditioning X Aging & 3921.929 & 2 & 1960.964 & 16.152 & $<0.001$ \\
\hline \multicolumn{6}{|l|}{ Chemical conditioning $X$} \\
\hline Aging & 4472.092 & 4 & 1118.023 & 9.209 & $<0.001$ \\
\hline Error & 43707.169 & 360 & 121.409 & & \\
\hline
\end{tabular}


Table 6 Means and standard deviations of $\mu$ TBS values in the different groups

\begin{tabular}{|c|c|c|c|c|}
\hline \multirow[b]{2}{*}{ Group } & \multicolumn{3}{|c|}{ Surface } & \multirow[b]{2}{*}{ Aged } \\
\hline & Subgroup & conditioning & Non-aged & \\
\hline & $\mathrm{SAL} / \mathrm{C}$ & No conditioning & $50.75(5.71) \mathrm{A}, \mathrm{a}$ & $37.41(7.32) \mathrm{B}, \mathrm{a}$ \\
\hline \multirow{2}{*}{ SAL } & SAL/UA & Universal adhesive & 62.02(9.78) A,b & 45.74(13.03) B,b \\
\hline & SAL/MM & $\gamma$-MPTS + 10-MDP & 74.49(11.67) A,c & 57.64(9.16) B,c \\
\hline \multirow{3}{*}{ GCO } & $\mathrm{GCO} / \mathrm{C}$ & No conditioning & 57.63(10.49) A,a & $40.65(10.59) \mathrm{B}, \mathrm{a}$ \\
\hline & $\mathrm{GCO} / \mathrm{UA}$ & Universal adhesive & 63.82(14.42) A,b & $56.34(10.25) \mathrm{B}, \mathrm{b}$ \\
\hline & $\mathrm{GCO} / \mathrm{MM}$ & $\gamma$-MPTS + 10-MDP & $82.02(11.81) \mathrm{A}, \mathrm{c}$ & 78.44(9.66) A,c \\
\hline \multirow{3}{*}{ RXU } & $\mathrm{RXU} / \mathrm{C}$ & No conditioning & $68.57(11.43) \mathrm{A}, \mathrm{a}$ & $35.68(8.13) \mathrm{B}, \mathrm{a}$ \\
\hline & RXU/UA & Universal adhesive & $74.89(9.88)$ A,a & $70.29(15.32) \mathrm{A}, \mathrm{b}$ \\
\hline & RXU/MM & $\gamma$-MPTS + 10-MDP & 76.49(11.47) A,a & $75.86(13.24) \mathrm{A}, \mathrm{b}$ \\
\hline
\end{tabular}

Values marked with the same upper-case letter were not significantly different within each row; values marked with the same lower-case letter were not significantly different within each column for each cement tested. 
Figure 1. Representative SEM images of the adherend surfaces of KC blocks. (A) and (B): subgroup $C$ at 300x and 1,000x; (C) and (D): subgroup $U A$ at 300x and 1,000x; (E) and (F): subgroup MM at 300x and 1,000x.
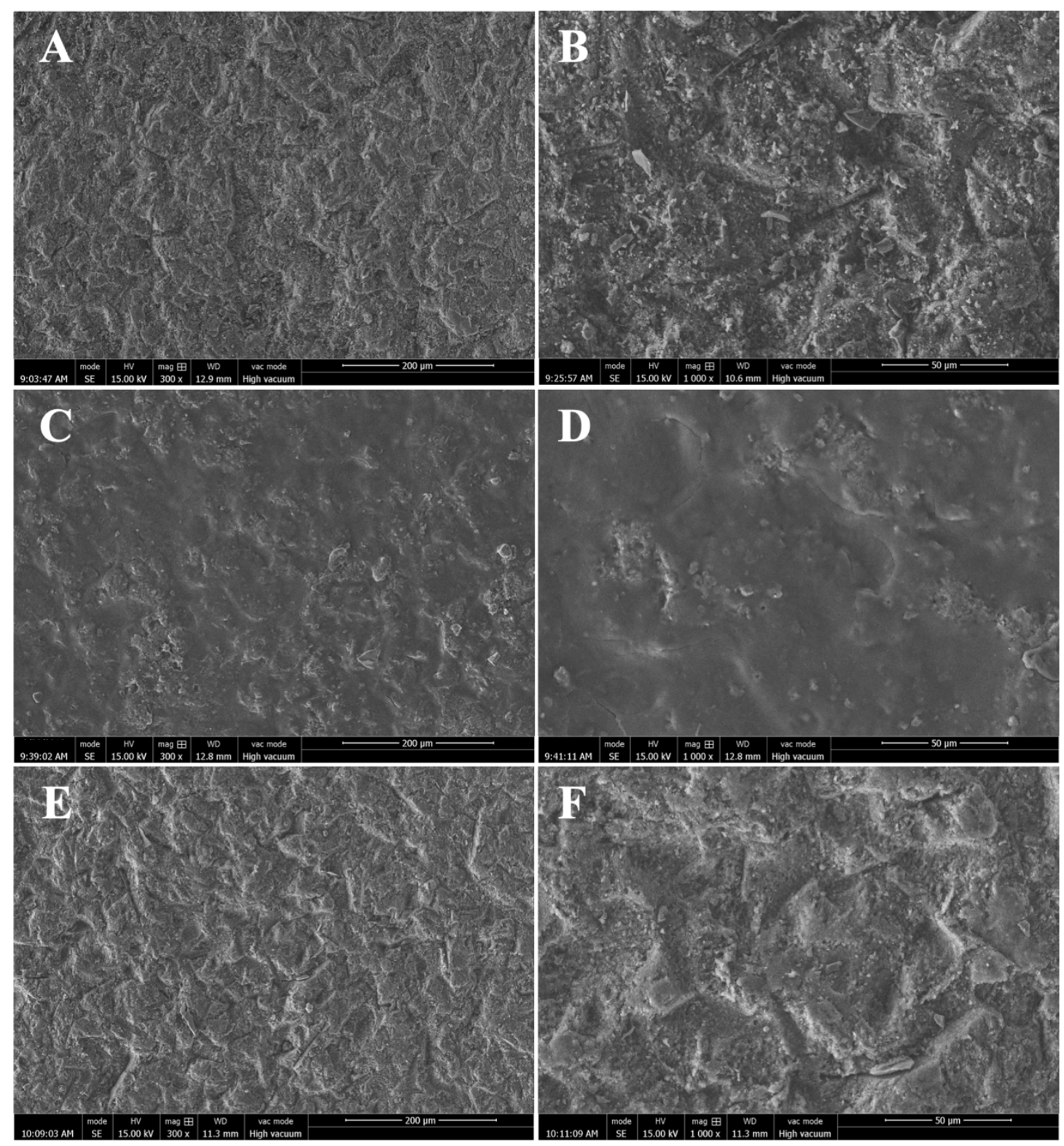
Figure 2. Failure mode distribution (percentage of each failure mode category) in the different groups
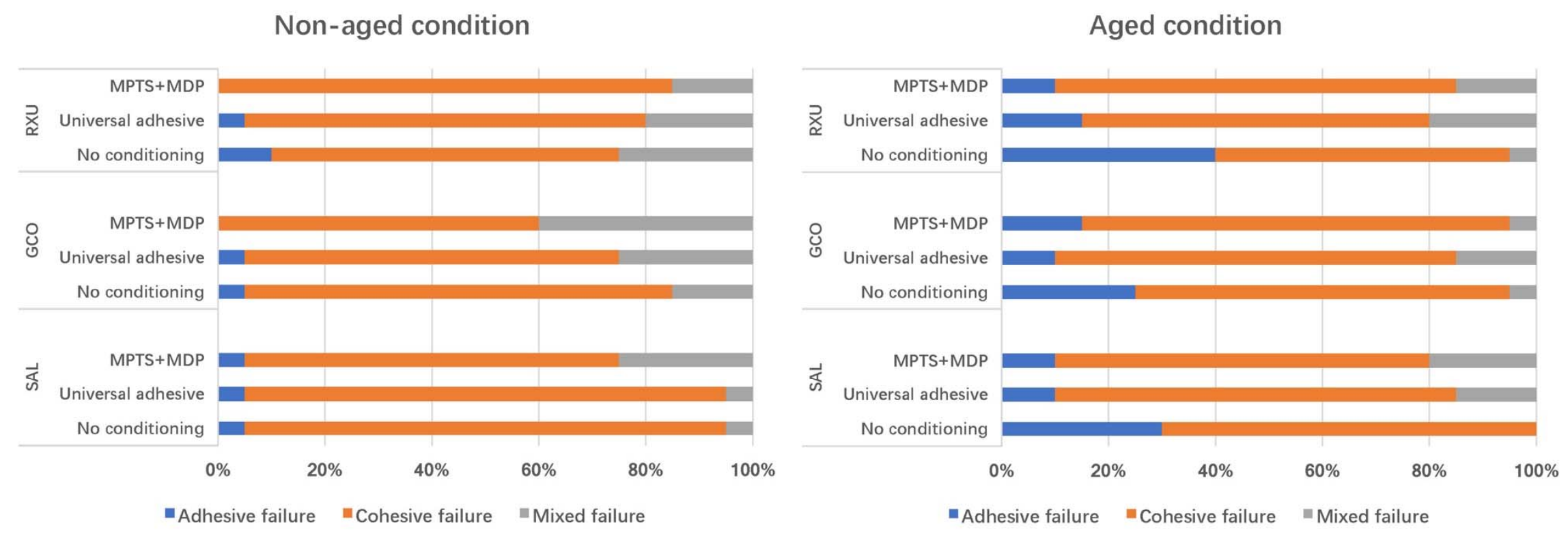